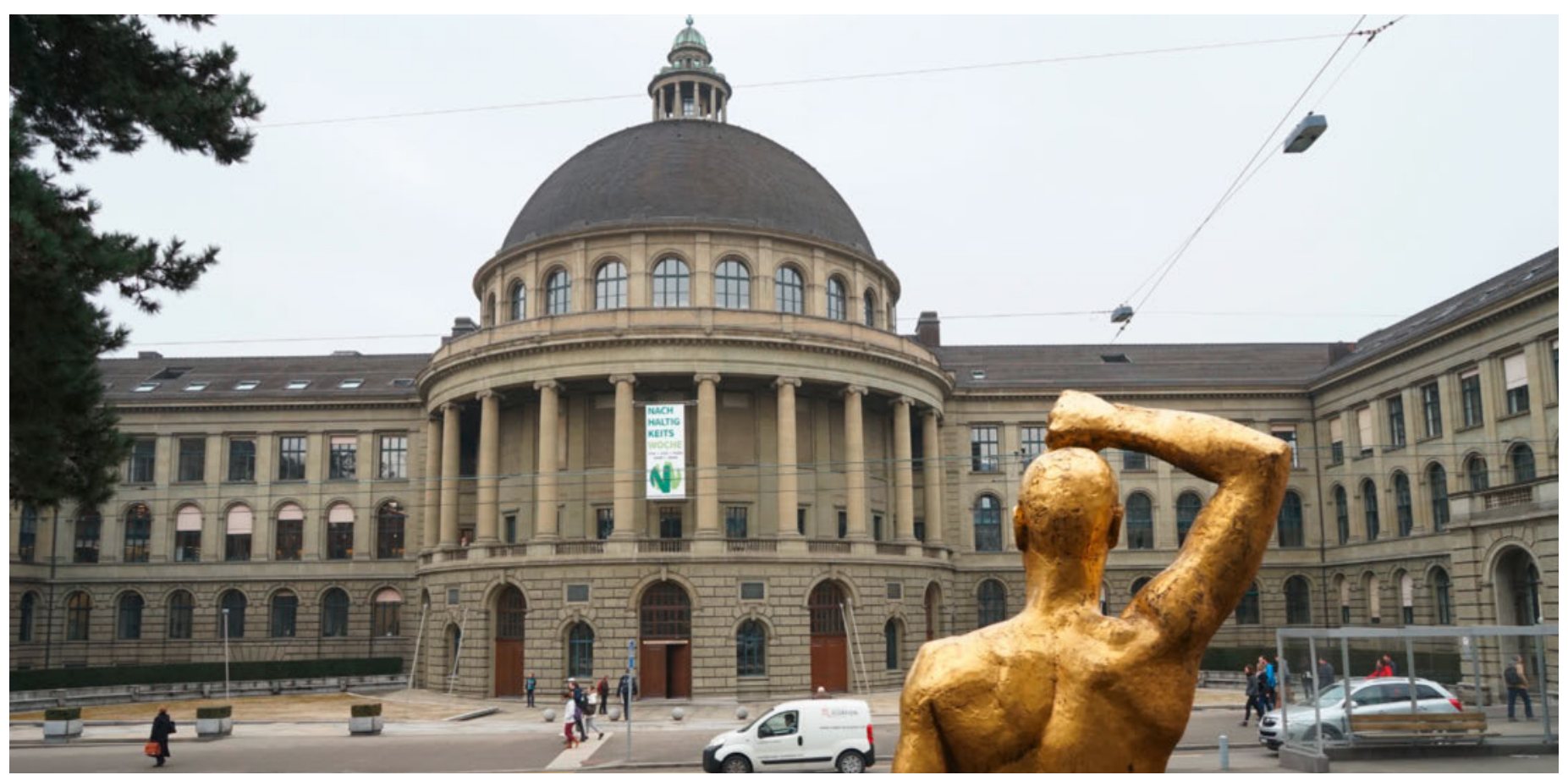

\title{
Das Bachelorstudium Humanmedizin an der ETH Zürich
}

\section{Jörg Goldhahn}

Prof. Dr. med., Leiter Projektteam Bachelor Humanmedizin

Im Jahr 2017 lief das Sonderprogramm «Humanmedizin» an. Dessen Ziel ist es, die Anzahl Absolventinnen und Absolventen eines Medizinstudiums von 865 im Jahr 2014 auf 1350 im Jahr 2025 zu erhöhen. Als direkte Reaktion auf dieses Sonderprogramm bietet die ETH Zürich seit letztem Herbst einen Studiengang zum Erreichen eines Bachelors in Humanmedizin an. Der Eintritt in das Masterstudium an einer Partneruniversität ist erstmals im Herbst 2020 möglich.

Bei sich stetig ändernden Lebensstilen und einer immer älter, aber auch dynamischer werdenden Gesellschaft kommt der Erhaltung und Verbesserung der Gesundheit eine zentrale Rolle zu. Gleichzeitig revolutionieren die Entwicklungen in der Bioinformatik, den molekularen Biowissenschaften und den bildgebenden Verfahren die Medizin. Um die neuen Möglichkeiten, beispielsweise der Medizintechnik oder der personalisierten Medizin, voll ausschöpfen zu können, braucht es künftig Ärztinnen und Ärzte mit fundiertem Wissen in mehreren Disziplinen, das heisst mit vertieften Kenntnissen in medizinischen wie auch in technischen Wissenschaften und in Naturwissenschaften.

Hier setzt der neue Bachelor Humanmedizin an der ETH Zürich an. Er vermittelt neben klassischen medizinischen Aspekten auch Themen aus dem medizintechnischen Bereich und gibt Einblicke in die digitale 


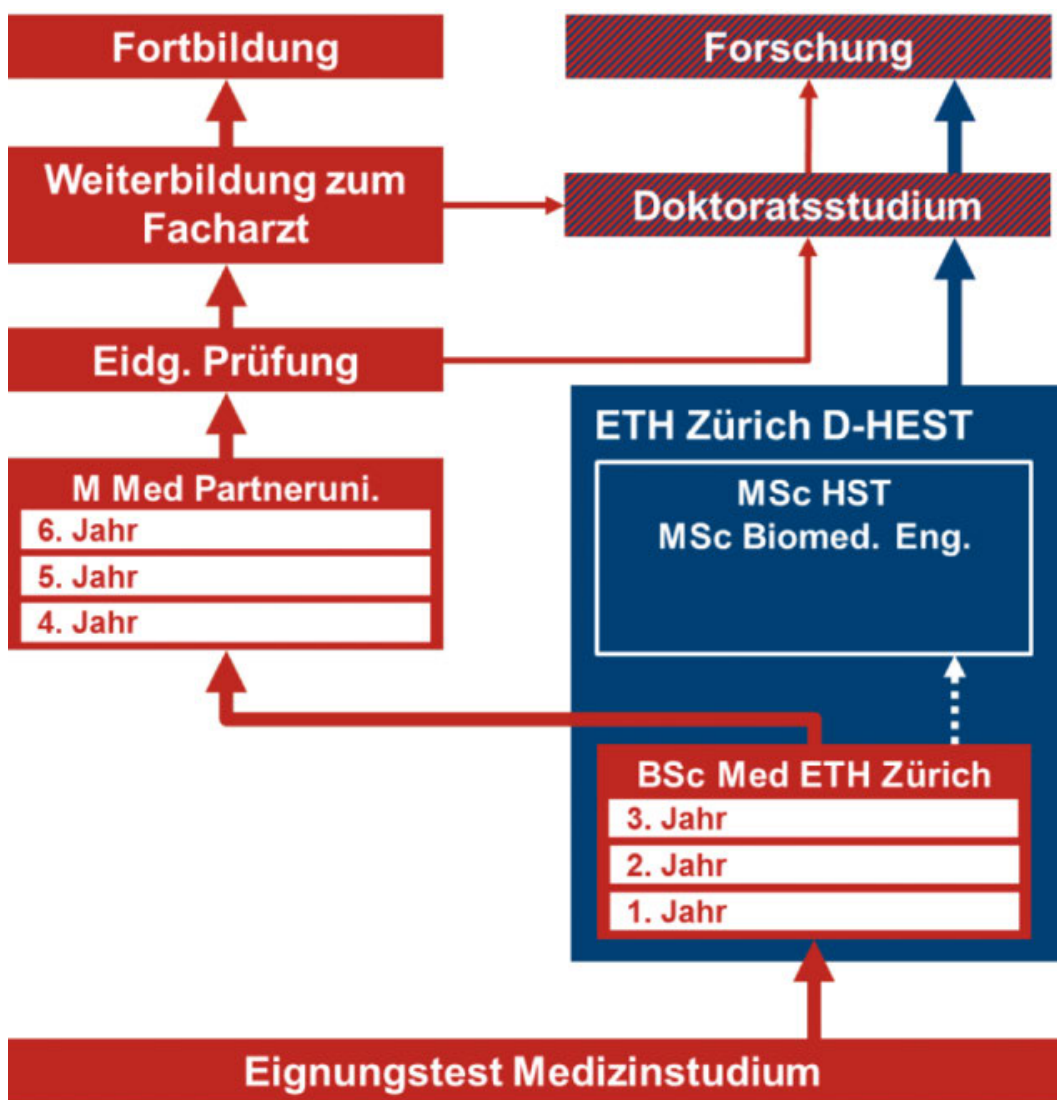

Aufbau des Studiums mit Forschungsoptionen für die Studierenden.

Korrespondenz:

Prof. Dr. med. Jörg Goldhahn Leiter Projektteam Bachelor Humanmedizin

Departement für Gesundheitswissenschaften D-HEST HCP H15.3

Leopold-Ruzicka-Weg 4 CH-8093 Zürich jgoldhahn[at]ethz.ch
Transformation der Medizin. Für das Masterstudium in Medizin wechseln die Bachelorabsolventen an eine Partneruniversität in der Schweiz.

\section{Details zum Studium}

Verantwortlich für den Bachelorstudiengang Humanmedizin an der ETH Zürich ist das Departement Gesundheitswissenschaften und Technologie (D-HEST). Das Medizinstudium ist in zwei Stufen aufgebaut:
Stufe 1: Das Bachelorstudium vermittelt die medizinischen und klinischen Grundlagen sowie Kenntnisse und Methoden in Mathematik, Naturwissenschaften und medizinischer Forschung. Diese erste Stufe wird mit dem Erwerb des Bachelordiploms abgeschlossen, das zum Eintritt in ein Masterstudium berechtigt.

Stufe 2: Das Masterstudium vermittelt die klinische Fachkompetenz und ermöglicht mit dem Erwerb des Masterdiploms den Zugang zur eidgenössischen Prüfung in Humanmedizin, welche die Berufsbefähigung darstellt. Das Masterstudium wird nicht von der ETH Zürich angeboten, sondern von drei Partneruniversitäten (UZH, UniBas, USI).

\section{Aufbau des Bachelorstudiums}

Das Studium besteht aus medizinischen, klinischen, naturwissenschaftlichen und medizinwissenschaftlichen Modulen. In den medizinischen Modulen werden nacheinander die einzelnen Organe und Organsysteme integrativ abgehandelt. Neben anatomischen und physiologischen Aspekten werden auch pathophysiologische, pharmakotherapeutische, klinische und wissenschaftliche Aspekte betrachtet. In den klinischen Modulen wird, in Zusammenarbeit mit den Partnern der ETH, insbesondere die (praktische) ArztPatient-Interaktion thematisiert. Diese Module ziehen sich durch das ganze Studium hindurch. Die naturwissenschaftlichen Fächer des ersten Jahres bilden die Grundlage für die Module, welche im zweiten und vor allem im dritten Studienjahr die Medizin mit der Wissenschaft verlinken. Ein translationales Forschungspraktikum, ETH intern oder bei Forschungspartnern, schliesst das Bachelorstudium ab.

Bildnachweis

Grafik ETHZ

Foto Matthias Scholer 